\title{
Influence of Temperature on the Interaction between Pd Clusters and the $\mathrm{TiO}_{2}(110)$ Surface
}

\author{
M. A. San-Miguel, ${ }^{*}$ J. Oviedo, and J.F. Sanz \\ Physical Chemistry Department, Faculty of Chemistry, University of Sevilla, clo Professor García González, 1. 41012, Sevilla, Spain
}

(Received 3 April 2007; published 6 August 2007)

\begin{abstract}
The behavior of a $\mathrm{Pd}$ nanocluster on the rutile $\mathrm{TiO}_{2}$ (110) surface has been analyzed by extensive first principles molecular dynamics simulations between $100 \mathrm{~K}$ and $1073 \mathrm{~K}$. Calculations predict a steep change in the morphological and electronic cluster structure around $800 \mathrm{~K}$ in excellent agreement with previous experimental evidence. At low temperature, the cluster geometry is mainly controlled by the substrate structure; however, upon the transition temperature, the cluster-substrate interaction decreases appreciably, and the cluster adopts a geometry more stable in vacuum and becomes metallic. These results illustrate at an atomistic level the influence of temperature on the geometrical and electronic properties of oxide-supported clusters.
\end{abstract}

Deposition of metals on metal oxide surfaces has been widely used in catalysis to obtain supports with specific properties [1]. This approach has found important applications in heterogeneous catalysis [2,3]. Among the metal oxide surfaces, rutile, and, in particular, the (110) face, has become a prototype due to its thermodynamic stability and relatively easy preparation [4]. Palladium clusters deposited on this surface can be considered as a catalyst model and have been extensively studied by different experimental techniques [5-9]. In the experiments, the surface is usually given some annealing treatment in which the role of temperature is especially important because on increasing the temperature, metal clusters can diffuse and coalesce. It is known that the size and distribution of metal clusters notably influence their catalytic activity [10]. In particular, photoelectron spectroscopy and resonant photoemission experiments for Pd coverage in the sub- and monolayer range on this surface [5] showed that annealing treatments at $700 \mathrm{~K}$ induced changes in the morphology of Pd clusters and the formation of islands, characterized by a high ratio between the volume and the area in contact with the substrate. It was also observed that their metallic character increased significantly upon the transition temperature.

Theoretical studies can provide valuable insights into the atomistic mechanisms of cluster deposition processes. Traditionally, these investigations used to be based on molecular dynamics (MD) simulations using semiempirical interatomic potentials [11,12]. However, most of these processes involve quantum effects that require a more precise description. First principles calculations on such large systems including dynamical effects are computationally demanding, and, until recently, they had been considered prohibitive. However, nowadays the development of modern fast computers makes this kind of calculations feasible $[13,14]$. Here, we report on density functional theory (DFT) calculations of the deposition of a 12 atoms $\mathrm{Pd}$ cluster on a nonstoichiometric $\mathrm{TiO}_{2}$ (110) surface. In order to analyze the evolution of the cluster properties as temperature increases, extensive first principles molecular dynamic simulations were carried out. We shall show that the theoretical analysis predicts a transition temperature in remarkably good agreement with experiments, and, in addition, it allows an atomistic interpretation for the changes in cluster properties.

In the present Letter, the implementation of DFT with plane waves has been used. The exchange-correlation contribution to the total energy was computed by means of the generalized gradient approximation (GGA) with the Perdew91 functional $[15,16]$. The inner electrons have been represented by the PAW (plane augmented waves) potentials [17-19]. One-electron states have been expanded in a plane wave basis with a $400 \mathrm{eV}$ kinetic energy cutoff. All calculations have been carried out at the $\Gamma$ point of the Brillouin zone and using the VASP (Vienna $a b$ initio simulation package) code [20-22]. A $(4 \times 2)$ supercell of three layers with optimized lattice parameters of $a=$ $4.616 \AA, c=2.974 \AA$, and $u=0.304$ has been used for the (110) surface. Full convergence tests and technical details can be found in a previous work [23].

Metal oxide surfaces generally contain a high density of point defects [24] which can play an important role in the catalytic activity. Specifically, in the rutile $\mathrm{TiO}_{2}$, the main point defects consist of bridging oxygen vacancies. By means of Scanning Tunnelling Microscopy (STM), the amount of oxygen vacancies has been quantified as 7$10 \%$ when the surface is annealed at $900 \mathrm{~K}[25,26]$. Point defects on oxide surfaces are considered preferential nucleation sites for cluster formation [27,28]. Recently, Sanz et al. [29] studied the adsorption of Pd atoms and dimers on the stoichiometric and defective $\mathrm{TiO}_{2}$ (110) surfaces and found that the adsorption on surface oxygen vacancies is stronger than that on the defect-free surface indicating that defects would act as nucleation sites. Our calculations were carried out on a defective surface created after removing a bridging oxygen atom from the system, which sets up a defect density of $12.5 \%$, close to the experimental value. 
First principles molecular dynamics simulations were performed every $100 \mathrm{~K}$ between 100 and $1073 \mathrm{~K}$. A typical simulation for a given temperature consisted of an equilibration run followed by a production run at the canonical ensemble. A time step of 3 femtoseconds was set up, and 1000 configurations were generated at each run. The last configuration generated was used as the first one at the next upper temperature. Each run required one week CPU time on a 24 nodes computer cluster (Pentium IV at $3 \mathrm{GHz}$ ).

For each temperature, the binding energy was estimated as

$$
E_{\text {bind }}=E_{12 \mathrm{Pd}-\mathrm{TiO}_{2}}-E_{\mathrm{TiO}_{2}}-E_{12 \mathrm{Pd}}
$$

where each term corresponds to optimized geometries. Figure 1 reflects two different regimes. Below the transition temperature (around $800 \mathrm{~K}$ ), the binding energy is approximately $1 \mathrm{eV}$ larger than at higher temperatures. This change might be related to the experimental behavior [5]. In order to investigate the origin of this transition temperature, the system structure was carefully analyzed at an atomistic level. Three snapshots at representative temperatures are shown in Fig. 2. At low temperature, the substrate structure controls the cluster geometry, which rearranges maximizing the interactions with bridging oxygen and with fivefold titanium atoms. This coordination mode is similar to that found for Pd dimers adsorbed on the surface [29]. On increasing temperature, the cluster atoms have enough kinetic energy to rearrange but keeping strong interaction with the substrate. Finally, at the highest temperature, a Pd atom in the first layer jumps up to the second layer, reducing thus the metal contact area. This fact would be the origin of the reduction in the binding energy observed at the transition temperature. Consequently, the cluster experiences smaller influence from the substrate and rearranges in another geometry more spherical shaped and similar to those found in gas phase. A larger cohesive energy for this cluster also reflects its higher stability.

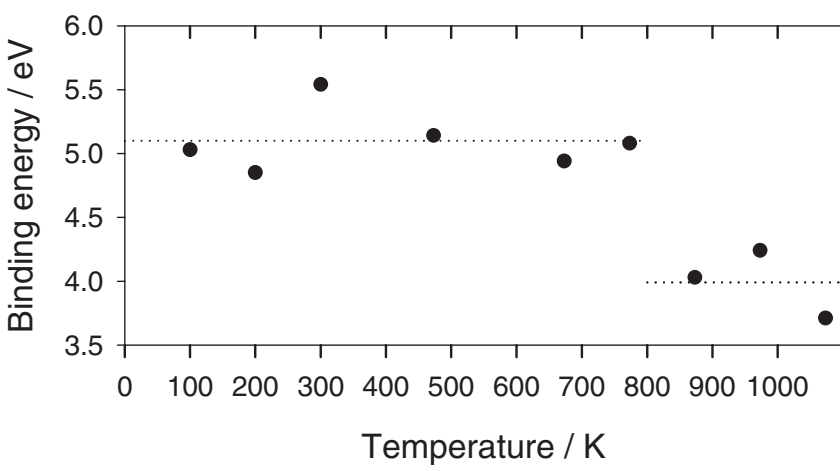

FIG. 1. Binding energy at several temperatures for a $12 \mathrm{Pd}$ atoms cluster deposited on a defective (110) rutile surface. Average values below and above the transition temperature $(800 \mathrm{~K})$ are shown by dotted lines.
Radial distribution functions (RDFs) are good indicators of structural changes (Fig. 3). The cluster transformation with temperature is clearly seen in Pd-Ti RDFs. The Pd-Ti distances reflect that at low temperature, the Pd atoms in the first layer are interacting strongly with fivefold $\mathrm{Ti}$ atoms. At higher temperatures, this peak diminishes and ends up disappearing almost completely. On the other hand, the Pd-Pd RDFs show in every case a single peak, which is centered at the Pd bulk distance $(2.78 \AA$ ) and the Pd-O RDFs show how distances widen by thermal agitation but with no significant changes.

The electronic structure was studied through the density of states. It is known that the creation of an oxygen vacancy in a stoichiometric surface causes a shift of the Fermi level from the top of the valence band to the bottom of the conduction band provoking a change in the electron conduction properties [4]. Upon one Pd atom deposition on the surface, the Fermi level remains at the same position although new features appear in the band gap. Furthermore, when the $\mathrm{Pd}_{12}$ cluster is adsorbed on the surface, the
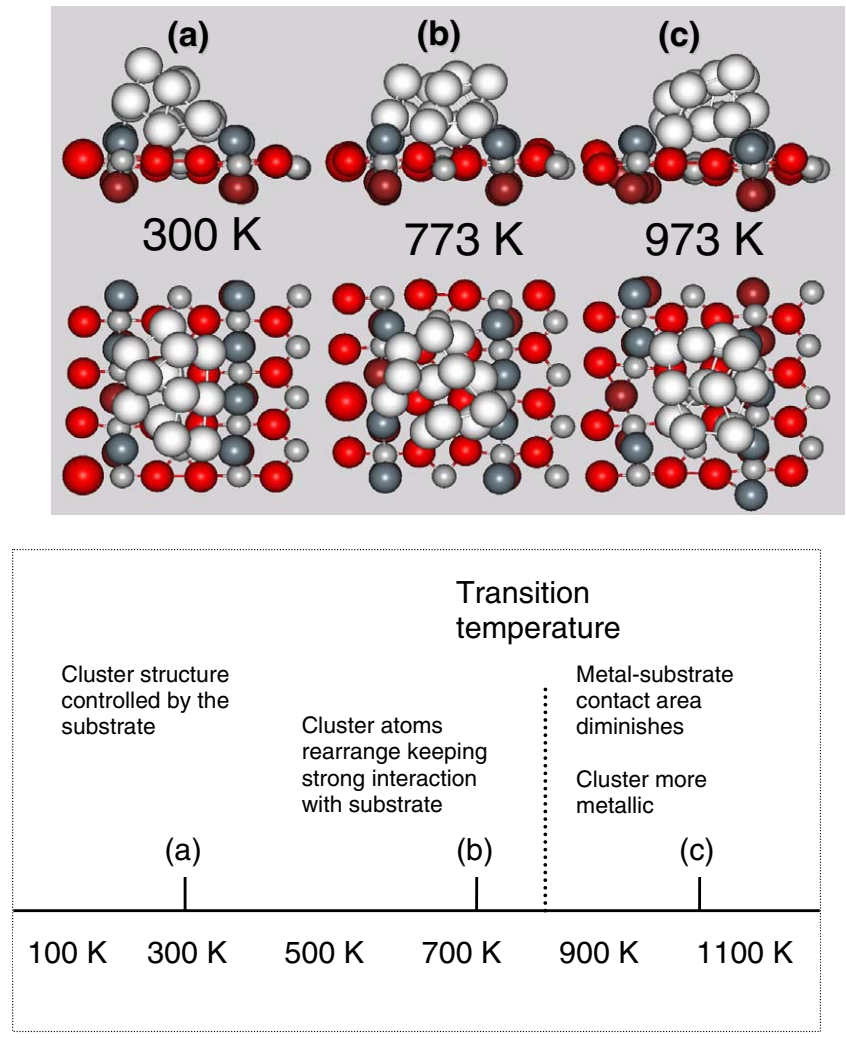

FIG. 2 (color online). Snapshots of a $12 \mathrm{Pd}$ atoms cluster deposited on a defective (110) rutile surface at three temperatures $(300,773$, and $973 \mathrm{~K})$. Side and top views are shown in the upper and lower panels, respectively. Ti atoms are represented by the smallest gray spheres, bridging $\mathrm{O}$ atoms by dark gray spheres, in-plane and sub-bridging atoms by black spheres, and Pd atoms by white spheres. Only the atoms in the outermost layers are depicted. 

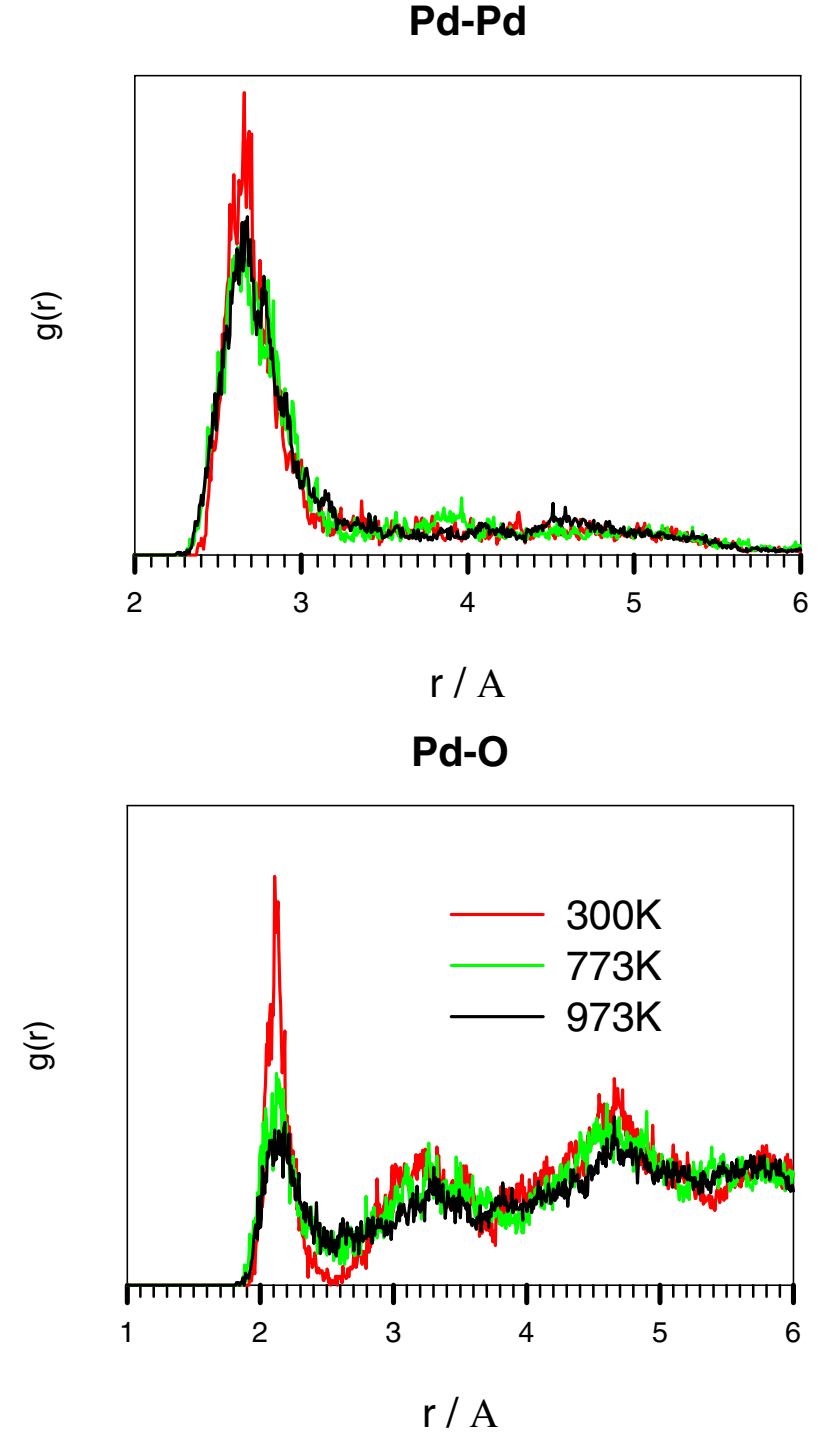

\section{Pd-Ti}

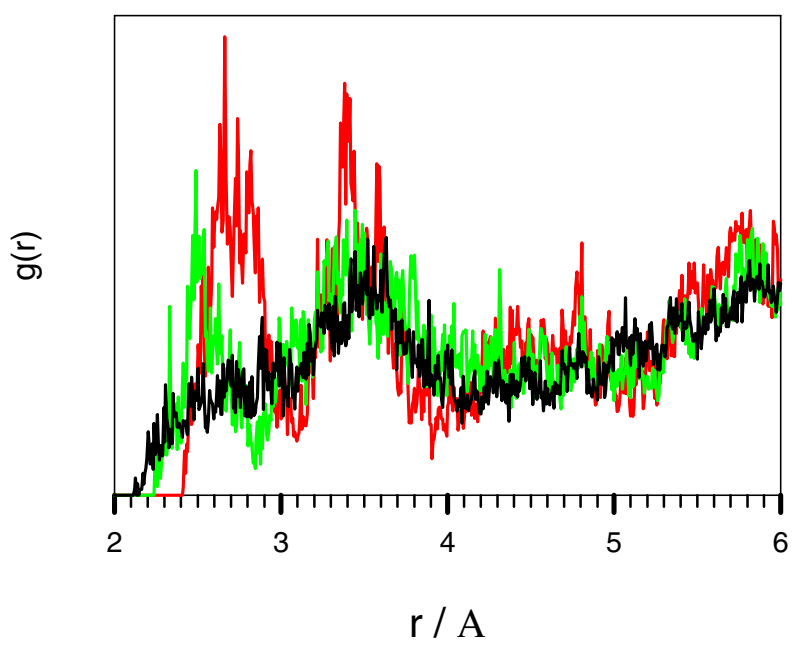

FIG. 3 (color online). Radial distribution functions for Pd-Pd, $\mathrm{Pd}-\mathrm{O}$, and $\mathrm{Pd}-\mathrm{Ti}$ species at the three temperatures discussed within the text. corresponding density of states plot shows that the band gap is fully filled by Pd electronic states, and this structure is affected negligibly by temperature. It is worth pointing out that the free $\mathrm{Pd}_{12}$ clusters resulting from our simulations have a metallic nature since their valence band is partially empty, independently of morphology and temperature.

It is particularly meaningful the analysis of the differences in electron density. They are computed through the formula

$$
\Delta \rho=\rho_{12 \mathrm{Pd}^{-\mathrm{TiO}_{2}}}-\rho_{\mathrm{TiO}_{2}}-\rho_{12 \mathrm{Pd}}
$$

where the electron densities of the surface and the Pd cluster are calculated on the geometries obtained from the optimization of the whole system. In this way, $\Delta \rho$ reflects how the electron density is localized in the space, compared to the isolated fragments, after the adsorption process. Thus, positive values would correspond to density gain and negative values to density loss. Figure 4 shows $\Delta \rho$ as surfaces of values of $\Delta \rho=+0.04 e$ [blue (dark gray)] and $\Delta \rho=-0.04 e$ [red (gray)]. At $300 \mathrm{~K}$, there is a wide blue area in the cluster, close to the support, which reveals an important polarization due to some charge transfer from the cluster to the surface. Although it is not possible for us to quantify the magnitude of such a transfer, it is clear that the cluster electronic structure is conditioned by the substrate. However, as temperature increases, the positive and negative areas become smaller, reflecting a stronger metallic behavior since the cluster electronic structure is not longer affected by the substrate. Particularly, at the highest temperature (i.e., $1073 \mathrm{~K}$ ), the charge is totally dispersed. This fact might be related to Della Negra's experiments, which indicated an increase in the cluster metallic behavior when the temperature is raised [5].

Extensive first principles molecular dynamics simulations based on the density functional theory have provided evidence of a cluster transformation around $800 \mathrm{~K}$, when a 12 atoms Pd cluster is annealed on the (110) rutile surface. The transition temperature is in good agreement with experimental observations, and its origin has been eluci-
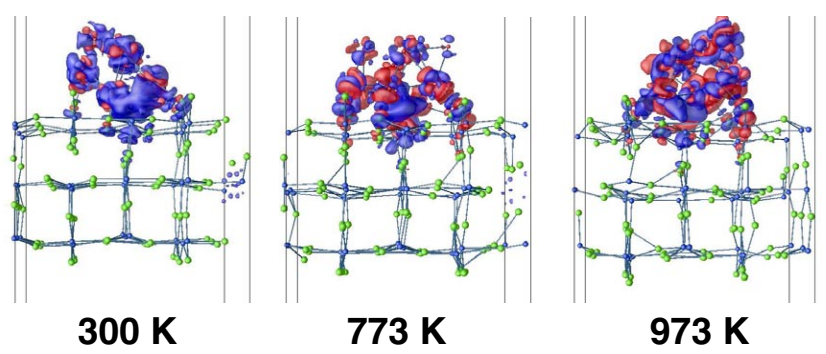

FIG. 4 (color online). Electron density difference maps for the system at three temperatures $(300 \mathrm{~K}, 773 \mathrm{~K}$, and $973 \mathrm{~K})$. Atoms are represented by small spheres. Red (gray) and blue (dark gray) clouds show negative and positive charge transfer, respectively. 
dated from an atomistic analysis. In the low temperature regime, the substrate controls strongly the cluster structure, which accommodates on the surface to maximize interactions with bridging oxygen and fivefold titanium atoms. Upon the transition temperature, the binding energy decreases by $1 \mathrm{eV}$ due to atoms moving from the first to the second layer. Consequently, the metal contact area with the substrate diminishes, and the cluster is more akin to the gas phase structure. Moreover, the cluster electronic properties change as temperature increases, and the cluster becomes more metallic.

This work was funded by the Spanish Ministerio de Educación y Ciencia, Project No. MAT2005-01872.

*Corresponding author: smiguel@us.es

[1] V. E. Henrich and P. A. Cox, The Surface Science of Metal Oxides (Cambridge University Press, Cambridge, 1994).

[2] C. T. Campbell, Surf. Sci. Rep. 27, 1 (1997).

[3] C. R. Henry, Surf. Sci. Rep. 31, 231 (1998).

[4] U. Diebold, Surf. Sci. Rep. 48, 53 (2003).

[5] M. Della Negra, N. M. Nicolaisen, Z. Li, and P. J. Møller, Surf. Sci. 540, 117 (2003).

[6] M. Bowker, P. Stone, R. Bennett, and N. Perkins, Surf. Sci. 497, 155 (2002).

[7] P. Stone, R. A. Bennett, S. Poulston, and M. Bowker, Surf. Sci. 433-435, 501 (1999).

[8] C. Xu, X. Lai, G. W. Zajac, and D. W. Goodman, Phys. Rev. B 56, 13464 (1997).

[9] T. Suzuki and R. Souda, Surf. Sci. 448, 33 (2000).

[10] M. Valden, X. Lai, and D. W. Goodman, Science 281, 1647 (1998).

[11] C.L. Cleveland and U. Landman, Science 257, 355 (1992).
[12] H. P. Cheng and U. Landman, Science 260, 1304 (1993).

[13] L. M. Molina and B. Hammer, Phys. Rev. Lett. 90, 206102 (2003).

[14] M. Moseler, H. Häkkinen, and U. Landmann, Phys. Rev. Lett. 89, 176103 (2002).

[15] J.P. Perdew, in Electronic Structure in Solids '91, edited by P. Ziesche and H. Eschrig (Akademie Verlag, Berlin, 1991).

[16] J.P. Perdew, J. A. Chevary, S. H. Vosko, K. A. Jackson, M. R. Pederson, D. J. Singh, and C. Fiolhais, Phys. Rev. B 46, 6671 (1992).

[17] M. C. Payne, M. P. Teter, D. C. Allan, T. A. Arias, and J. D. Joannopoulos, Rev. Mod. Phys. 64, 1045 (1992).

[18] P. E. Blöchl, Phys. Rev. B 50, 17953 (1994).

[19] G. Kresse and J. Joubert, Phys. Rev. B 59, 1758 (1999).

[20] G. Kresse and J. Hafner, Phys. Rev. B 47, 558 (1993).

[21] G. Kresse and J. Furthmüller, Comput. Mater. Sci. 6, 15 (1996).

[22] G. Kresse and J. Furthmüller, Phys. Rev. B 54, 11169 (1996).

[23] J. Oviedo, M. A. San Miguel, and J.F. Sanz, J. Chem. Phys. 121, 7427 (2004).

[24] C. Barth and C. R. Henry, Phys. Rev. B 91, 196102 (2003).

[25] U. Diebold, J. Lehman, T. Mahmoud, M. Kuhn, G. Leonardelli, W. Hebenstreit, M. Schmid, and P. Varga, Surf. Sci. 411, 137 (1998).

[26] E. L. D. Hebenstreit, W. Hebestreit, and U. Diebold, Surf. Sci. 461, 87 (2000); 470, 347 (2001).

[27] L. Giordano, C. Di Valentin, J. Goniakowski, and G. Pacchioni, Phys. Rev. Lett. 92, 096105 (2004).

[28] L. Xu, G. Henkelman, C. T. Campbell, and H. Jónsson, Phys. Rev. Lett. 95, 146103 (2005).

[29] J.F. Sanz and A. Márquez, J. Phys. Chem. C 111, 3949 (2007). 